\title{
Automated Model-Based Bias Field Correction of MR Images of the Brain
}

\author{
Koen Van Leemput,* Frederik Maes, Dirk Vandermeulen, and Paul Suetens
}

\begin{abstract}
We propose a model-based method for fully automated bias field correction of MR brain images. The MR signal is modeled as a realization of a random process with a parametric probability distribution that is corrupted by a smooth polynomial inhomogeneity or bias field. The method we propose applies an iterative expectation-maximization (EM) strategy that interleaves pixel classification with estimation of class distribution and bias field parameters, improving the likelihood of the model parameters at each iteration. The algorithm, which can handle multichannel data and slice-by-slice constant intensity offsets, is initialized with information from a digital brain atlas about the $a$ priori expected location of tissue classes. This allows full automation of the method without need for user interaction, yielding more objective and reproducible results. We have validated the bias correction algorithm on simulated data and we illustrate its performance on various MR images with important field inhomogeneities. We also relate the proposed algorithm to other bias correction algorithms.
\end{abstract}

Index Terms-Bias field, digital brain atlas, MRI, tissue classification.

\section{INTRODUCTION}

A CCURATE segmentation of magnetic resonance (MR) images of the brain is of interest in the study of many brain disorders. In multiple sclerosis, for instance, quantification of white matter lesions is necessary for drug treatment assessment, while in schizophrenia and epilepsy, volumetry of gray matter, white matter, and cerebro-spinal fluid is important to characterize morphological differences between subjects. Since such studies typically involve vast amounts of data, manual segmentation is too time consuming. Moreover, such manual segmentations show large inter- and intraobserver variability. Hence, there is a need for automated segmentation tools.

Manuscript received September 11, 1998; revised July 9, 1999. This work was supported by the EC-funded BIOMED-2 program under Grant BMH4CT96-0845 (BIOMORPH), by the Research Fund KULeuven under Grant GOA/99/05 (VHS+), and by the EC-funded BIOMED-2 program under Grant BMH4-CT98-6048 (QAMRIC). The work of D. Vandermeulen was supported in part by the Fund for Scientific Research-Flanders (Belgium) (FWO-Vlaanderen) under Grant 1.5.397.97. The work of K. Van Leemput was supported in part by a grant from the Flemish Institute for the Improvement of the Scientific-Technological Research in the Industry (IWT). F. Maes is a Postdoctoral Fellow of Research for the Fund for Scientific ResearchFlanders (Belgium) (FWO-Vlaanderen). The Associate Editors responsible for coordinating the review of this paper and recommending its publication were M. Viergever and W. Niessen. Asterisk indicates corresponding author.

*K. Van Leemput, F. Maes, D. Vandermeulen, and P. Suetens are with the group of Medical Image Computing (Radiology-ESAT/PSI), Faculties of Medicine and Engineering, University Hospital Gasthuisberg, Herestraat 49, B-3000 Leuven, Belgium.

Publisher Item Identifier S 0278-0062(99)09633-0.
A major problem for automated MR image segmentation is the corruption with a smoothly varying intensity inhomogeneity or bias field [1], [2]. This bias is inherent to MR imaging and is caused by equipment limitations and patient-induced electrodynamic interactions [2]. Although not always visible for a human observer, such a bias can cause serious misclassifications when intensity-based segmentation techniques are used. Correcting the MR image intensities for bias field inhomogeneity is therefore a necessary requirement for robust automated segmentation.

Early methods for bias field estimation and correction used phantoms to empirically measure the bias field inhomogeneity [3]. However, this approach assumes that the bias field is patient independent, which it is not [2]. Furthermore, it is required that the phantom's scan parameters are the same as the patient's, making this technique impractical and even useless as a retrospective bias correction method. In a similar vein, bias correction methods have been proposed for surface coil MR imaging, using an analytic correction of the MR antenna reception profile [4], but these suffer from the same drawbacks as phantom-based methods. Another approach, using homomorphic filtering [5], assumes that the frequency spectrum of the bias field and the image structures are well separated, but this assumption is generally not valid for MR images [3], [6].

While bias field correction is needed for good segmentation, many approaches have exploited the idea that a good segmentation helps to estimate the bias field. Dawant et al. [6] manually selected some points inside white matter and estimated the bias field as the least-squares spline fit to the intensities of these points. They also presented a slightly different version where the reference points are obtained by an intermediate classification operation, using the estimated bias field for final classification. Meyer et al. [7] also estimated the bias field from an intermediate segmentation, but they allowed a region of the same tissue type to be broken up into several subregions, which creates additional but sometimes undesired degrees of freedom.

Wells et al. [8] described an iterative method that interleaves classification with bias field correction based on maximum likelihood parameter estimation using the expectationmaximization (EM) algorithm [9]. However, for each set of similar scans to be processed their method, as well as its refinement by other authors [10], [11], needs to be supplied with specific tissue class conditional intensity models. Such models are typically constructed by manually selecting representative points of each of the classes considered, which may result in 
segmentations that are not fully objective and reproducible.

In contrast, the method we present here does not require such a preceding training phase. Instead, we use a digital brain atlas with a priori probability maps for each tissue class to automatically construct intensity models for each individual scan being processed. This results in a fully automated algorithm that interleaves classification, bias field estimation, and estimation of class-conditional intensity distribution parameters.

In this paper we focus on the bias correction component of the algorithm. We present validation of the bias correction on simulated data with known ground truth and demonstrate that the algorithm is capable of robustly estimating the bias of both single-channel and multichannel MR images. In the companion paper [12], we focus on tissue classification, which we evaluate by comparison with interactive segmentations by a trained expert. In this second paper [12] we also extend the current approach to include contextual constraints and investigate the use of Markov random fields to improve the segmentation generated by the algorithm.

This paper is organized as follows. Our bias correction algorithm is explained in detail in Section II. Validation on simulated data is presented in Section III and the performance of the method on single and multichannel MR data is illustrated in Section IV. The main characteristics of the algorithm are discussed and compared to other approaches in Section V. Finally, a summary and some conclusions are given in Section VI.

\section{METHOD}

Our approach for estimating the bias field and the tissue classification of an MR image is based on maximum likelihood parameter estimation using the EM algorithm. We first briefly discuss a two-step EM algorithm for MRI tissue classification without bias correction, which iteratively alternates between estimation of intensity model parameters and tissue classification, maximizing the likelihood of the model parameters at each iteration. We then extend this to a three-step procedure by including a bias correction step such that the algorithm now alternates between estimation of intensity model parameters, tissue classification, and bias field estimation. We also extend the algorithm to include multichannel input data and to cope with background noise and slice-by-slice constant offsets.

\section{A. Two-Step Classification Algorithm Without Bias Correction}

The EM algorithm (Dempster et al. [9]) is a general technique for finding maximum likelihood parameter estimates in problems with missing data. It is assumed that the observed data are only part of the underlying complete data. The EM algorithm tries to find the maximum likelihood parameter estimates for the observed data by estimating the missing data, based on the current parameter estimation. Combined with the observed data, this yields an estimation of the complete data. The EM algorithm then estimates the maximum likelihood for the observed data by maximizing the likelihood for the estimated complete data.

Applied to the problem of MRI tissue classification, the observed data are the signal intensities, the missing data are the classification of the images, and the parameters are classconditional intensity distribution parameters. It is assumed that each voxel value is selected at random from one of $K$ classes. The EM algorithm then interleaves class-conditional distribution parameter estimation and a statistical classification of the image voxels into $K$ classes.

Modeling each class by a normal distribution $G_{\sigma}(y-\mu)$ with mean $\mu$ and variance $\sigma^{2}$, as in [8] and [13], the probability density that class $j$ has generated the voxel value $y_{i}$ at position $i$ is

$$
p\left(y_{i} \mid \Gamma_{i}=j, \theta_{j}\right)=G_{\sigma_{j}}\left(y_{i}-\mu_{j}\right)
$$

with $\Gamma_{i} \in\{j \mid j=1 \cdots K\}$ the tissue class at position $i$ and $\theta_{j}=\left\{\mu_{j}, \sigma_{j}\right\}$ the distribution parameters for class $j$. Defining $\theta=\left\{\theta_{j}\right\}$ as the model parameters, the overall probability density for $y_{i}$ is

$$
p\left(y_{i} \mid \theta\right)=\sum_{j} p\left(y_{i} \mid \Gamma_{i}=j, \theta_{j}\right) p\left(\Gamma_{i}=j\right)
$$

which is a mixture of normal distributions. Since all the voxel intensities are assumed to be statistically independent, the probability density for the image $y$ given the model is

$$
p(y \mid \theta)=\prod_{i} p\left(y_{i} \mid \theta\right) .
$$

The maximum likelihood estimates for the parameters $\mu_{j}$ and $\sigma_{j}$ can be found by maximization of $p(y \mid \theta)$, equivalent to minimization of $-\Sigma_{i} \log _{e}\left(p\left(y_{i} \mid \theta\right)\right)$. The expression for $\mu_{j}$ is given by the condition that

$$
\frac{\partial}{\partial \mu_{j}}\left(-\sum_{i} \log _{e}\left(\sum_{j} p\left(y_{i} \mid \Gamma_{i}=j, \theta_{j}\right) p\left(\Gamma_{i}=j\right)\right)\right)=0 .
$$

Differentiating and substituting $p\left(y_{i} \mid \Gamma_{i}=j, \theta_{j}\right)$ by the Gaussian distribution (1) yields

$$
\sum_{i} p\left(\Gamma_{i}=j \mid y_{i}, \theta\right)\left(y_{i}-\mu_{j}\right)=0
$$

where Bayes' rule was used

$$
p\left(\Gamma_{i}=j \mid y_{i}, \theta\right)=\frac{p\left(y_{i} \mid \Gamma_{i}=j, \theta_{j}\right) p\left(\Gamma_{i}=j\right)}{\sum_{j} p\left(y_{i} \mid \Gamma_{i}=j, \theta_{j}\right) p\left(\Gamma_{i}=j\right)} .
$$

Rearranging the terms yields the expression for $\mu_{j}$ :

$$
\mu_{j}=\frac{\sum_{i} y_{i} p\left(\Gamma_{i}=j \mid y_{i}, \theta\right)}{\sum_{i} p\left(\Gamma_{i}=j \mid y_{i}, \theta\right)} .
$$

The same approach can be followed to derive the expression for $\sigma_{j}$ :

$$
\sigma_{j}^{2}=\frac{\sum_{i} p\left(\Gamma_{i}=j \mid y_{i}, \theta\right)\left(y_{i}-\mu_{j}\right)^{2}}{\sum_{i} p\left(\Gamma_{i}=j \mid y_{i}, \theta\right)} .
$$

Note that (2) performs a classification, whereas (3) and (4) are parameter estimates. Together they form a set of coupled 
equations, which can be solved numerically by alternately iterating between classification (step 1) and parameter estimation (step 2). The algorithm fills in the missing data during step 1 and then finds the parameters that maximize the likelihood for the complete data during step 2. It has been shown in [14] that the likelihood is guaranteed to increase at each iteration for such an algorithm.

\section{B. Three-Step Classification Algorithm with Bias Correction}

To correct for bias field inhomogeneities during classification, we propose to extend this two-step classification algorithm with a bias field estimation step. Each class $j$ is modeled by a normal distribution as in the original algorithm, but we now include a parametric model for the bias field. We model the inhomogeneity as a linear combination $\Sigma_{k} c_{k} \phi_{k}(x)$ of smooth basis functions $\phi_{k}(x)$. In our implementation, we used polynomial basis functions, but the theory is valid for any kind of smooth basis functions, such as splines. Field inhomogeneities are known to be multiplicative. We therefore first logarithmically transform the intensities in order to make the bias additive. Our model is then

$$
p\left(y_{i} \mid \Gamma_{i}=j, \theta_{j}, C\right)=G_{\sigma_{j}}\left(y_{i}-\mu_{j}-\sum_{k} c_{k} \phi_{k}\left(x_{i}\right)\right)
$$

and

$$
p\left(y_{i} \mid \theta, C\right)=\sum_{j} p\left(y_{i} \mid \Gamma_{i}=j, \theta_{j}, C\right) p\left(\Gamma_{j}=j\right)
$$

with $C=\left\{c_{k}\right\}$ the bias field parameters. Bias field correction thus involves finding the parameters $\mu_{j}, \sigma_{j}$ and $c_{k}$ that maximize the likelihood $\Pi_{i} p\left(y_{i} \mid \theta, C\right)$.

Following the same approach as for (3) and (4), the expressions for the distribution parameters $\mu_{j}$ and $\sigma_{j}$ are

$$
\mu_{j}=\frac{\sum_{i} p\left(\Gamma_{i}=j \mid y_{i}, \theta, C\right)\left(y_{i}-\sum_{k} c_{k} \phi_{k}\left(x_{i}\right)\right)}{\sum_{i} p\left(\Gamma_{i}=j \mid y_{i}, \theta, C\right)}
$$

and

$$
\sigma_{j}^{2}=\frac{\sum_{i} p\left(\Gamma_{i}=j \mid y_{i}, \theta, C\right)\left(y_{i}-\mu_{j}-\sum_{k} c_{k} \phi_{k}\left(x_{i}\right)\right)^{2}}{\sum_{i} p\left(\Gamma_{i}=j \mid y_{i}, \theta, C\right)} .
$$

Equations (5) and (6) are similar to (3) and (4) of the twostep EM algorithm. The only difference is that the data is corrected for a bias field before the distribution parameters are calculated.

Setting the partial derivative for $c_{k}$ of $-\Sigma_{i} \log _{e}\left(p\left(y_{i} \mid \theta, C\right)\right)$ to zero yields

$$
\begin{aligned}
\sum_{i} \phi_{k}\left(x_{i}\right) & \left(\sum_{j} \frac{p\left(\Gamma_{i}=j \mid y_{i}, \theta, C\right)}{\sigma_{j}^{2}}\right. \\
& \left.\cdot\left[y_{i}-\mu_{j}-\sum_{l} c_{l} \phi_{l}\left(x_{i}\right)\right]\right)=0 \quad \forall k .
\end{aligned}
$$

Solving this equation for $\left\{c_{l}\right\}$ does not seem very tractable, but combining all equations for all $k$ and introducing matrix notation simplifies the problem considerably

$$
C=\left[\begin{array}{c}
c_{1} \\
c_{2} \\
\vdots
\end{array}\right]=\left(A^{T} W A\right)^{-1} A^{T} W R
$$

with

$$
\begin{aligned}
A & =\left[\begin{array}{cccc}
\phi_{1}\left(x_{1}\right) & \phi_{2}\left(x_{1}\right) & \phi_{3}\left(x_{1}\right) & \cdots \\
\phi_{1}\left(x_{2}\right) & \phi_{2}\left(x_{2}\right) & \phi_{3}\left(x_{2}\right) & \cdots \\
\vdots & \vdots & \vdots & \ddots
\end{array}\right] \\
W & =\operatorname{diag}\left(w_{i}\right), \quad w_{i}=\sum_{j} w_{i j}, \\
w_{i j} & =\frac{p\left(\Gamma_{i}=j \mid y_{i}, \theta, C\right)}{\sigma_{j}^{2}} \\
R & =\left[\begin{array}{c}
y_{1}-\tilde{y}_{1} \\
y_{2}-\tilde{y}_{2} \\
\vdots
\end{array}\right], \quad \tilde{y}_{i}=\frac{\sum_{j} w_{i j} \mu_{j}}{\sum_{j} w_{i j}} .
\end{aligned}
$$

The matrix $A$ represents the geometry of the bias field model, each of its columns evaluating one basis function $\phi_{k}$ at all coordinates $x_{i} . W$ is a diagonal matrix of voxel weights $w_{i}, w_{i}$ being the sum over all classes of the inverse of their variance weighted by the class probability of voxel $i$. The vector $R$ represents the residue image, i.e., the difference between the original data $y$ and the estimated bias-corrected data $\tilde{y}$. Equation (7) is a weighted least-squares fit. From the intermediate classification and Gaussian distribution parameter estimates, a prediction of the signal without bias is constructed and subtracted from the original data. Each voxel in the resulting residue image is assigned a weight, inversely proportional to its weighted variance. The bias field is obtained as the weighted least-squares fit to the residue image.

To get more insight into (7), consider the special case in which each voxel is exclusively assigned to a single class. The predicted signal $\tilde{y}$ is then equal to each pixel's class mean and the weights $w_{i}$ are inversely proportional to the variance of that class. Another case in which (7) simplifies is when all the variances $\sigma_{j}^{2}$ are the same. The weights are then all the same as well, and the predicted signal is the class likelihood weighted sum of the means $\mu_{j}$ of all classes.

Iterating between (2) and (5)-(7), the algorithm interleaves classification (step 1), class distribution parameter estimation (step 2), and bias estimation (step 3). The algorithm fills in the missing data during step 1 and then finds the parameters that increase the likelihood for the complete data during steps 2 and 3. While the two-step classification algorithm of Section II-A iterates between steps 1 and 2 and does not include bias corrections, the method of Wells et al. [8] applies steps 1 and 3, but does not update the class distribution parameters during iterations. Since in steps 2 and 3 of our algorithm the likelihood is only increased but not maximized, our algorithm is called a generalized EM algorithm (GEM) [9]. It has been shown in [14] that likelihood is guaranteed to increase at each iteration for such GEM algorithms. 
To start the iterative scheme presented above, we need an initial estimate of the class distribution parameters or of the tissue classification itself. Instead of having the user manually indicate pixels that are representative for each class, as Wells et al. [8] do, we use a digital brain atlas that provides a priori tissue probabilities for each voxel in the image. This will be discussed in Section II-D.

\section{Further Extensions}

The algorithm, as presented so far, can only handle singlechannel input data. Since each of the channels of multispectral MR images provides additional information, we extend the algorithm to multichannel data in order to improve the classification. Further extensions include a model for the background noise and compensation for slice-by-slice constant intensity offsets.

1) Multichannel Input Data: For multichannel data $Y=$ $\left[y_{1} y_{2} \cdots\right]$, the Gaussian distributions of the classes $j$ become multivariate normals with mean $\boldsymbol{\mu}_{j}$ and covariance matrix $\Sigma_{j}$. Extension of (3) and (4) to multivariate normals is straightforward (see, for instance, [15]). Each channel has its own polynomial bias field. Equation (7) for the bias field parameters now becomes

$$
\begin{gathered}
{\left[\begin{array}{ccc}
A^{T} W_{11} A & A^{T} W_{12} A & \cdots \\
A^{T} W_{21} A & A^{T} W_{22} A & \cdots \\
\vdots & \vdots & \ddots
\end{array}\right]\left[\begin{array}{c}
C_{1} \\
C_{2} \\
\vdots
\end{array}\right]} \\
\quad=\left[\begin{array}{c}
A^{T}\left(W_{11} R_{11}+W_{12} R_{12}+\cdots\right) \\
A^{T}\left(W_{21} R_{21}+W_{22} R_{22}+\cdots\right) \\
\vdots
\end{array}\right]
\end{gathered}
$$

with $C_{l}$ the vector of bias field polynomial coefficients of channel $l, W_{k l}$ the weight matrix for the pair of channels $k$ and $l$, and $R_{k l}$ the corresponding residue image

$$
\begin{gathered}
W_{k l}=\operatorname{diag}\left(w_{i}^{k l}\right), \quad R_{k l}=y_{l}-\frac{\sum_{j} w_{i j}^{k l}\left[\boldsymbol{\mu}_{j}\right]_{l}}{\sum_{j} w_{i j}^{k l}} \\
w_{i}^{k l}=\sum_{j} w_{i j}^{k l}, \quad w_{i j}^{k l}=p\left(\Gamma_{i}=j \mid y_{i}, \theta, C\right)\left[\Sigma_{j}^{-1}\right]_{k l} .
\end{gathered}
$$

Each element in the weight matrix $W_{k l}$ is a weighted sum over all classes of the elements $\left[\boldsymbol{\Sigma}_{j}^{-1}\right]_{k l}$ of the inverse of the class covariance matrices. Thus, the bias field of the different channels cannot be calculated separately in the general case. If all the covariance matrices $\boldsymbol{\Sigma}_{j}$ were diagonal, the $W_{k l}$ would be zero for $k \neq l$, which means that the bias fields are uncoupled. Solving (8) would then be the same as solving the single-channel equation (7) for each channel separately.

2) Background Model: The image background requires a special treatment since there is no signal except for the noise. Hence, the background is not affected by the bias field and should therefore not constrain the polynomial bias estimation.

The background noise can be included explicitly in the model. In [16], it is shown that in image regions with no MR signal, the noise is governed by the Rayleigh distribution. Since we work with the logarithm of the images, the distribution becomes

$$
p_{b}\left(y \mid \sigma_{b}\right)=\frac{e^{2 y}}{\sigma_{b}^{2}} e^{-e^{2 y} / 2 \sigma_{b}^{2}}
$$

with $y$ the measured pixel intensity and $\sigma_{b}^{2}$ the variance parameter of the background noise. The model now becomes

$$
\begin{aligned}
p\left(y_{i} \mid \theta, C, \sigma_{b}\right)= & p_{b}\left(y_{i} \mid \sigma_{b}\right) p\left(\Gamma_{i}=b\right) \\
& +\sum_{j \neq b} p\left(y_{i} \mid \Gamma_{i}=j, \theta_{j}, C\right) p\left(\Gamma_{i}=j\right)
\end{aligned}
$$

with $b$ the background class. In this model the tissue classes are affected by the bias field, while the background is not. Using the same approach as before to find the parameters maximizing the likelihood, the expression for the variance parameter of the noise in the background becomes

$$
\sigma_{b}^{2}=\frac{\sum_{i} p\left(\Gamma_{i}=b \mid y_{i}, \theta, C, \sigma_{b}\right) e^{2 y_{i}}}{2 \sum_{i} p\left(\Gamma_{i}=b \mid y_{i}, \theta, C, \sigma_{b}\right)} .
$$

In case of multichannel input data, this expression can be used for each channel separately, since the noise in the different channels is independent. The other equations remain exactly the same, except (7) for the bias correction. The bias is now only estimated with respect to the nonbackground classes. Pixels assigned to the background have a zero weight for the bias polynomial fit, which comes as a natural result since the background was modeled to be unaffected by the bias field.

3) Slice-by-Slice Constant Offsets: In addition to a smoothly varying field inhomogeneity, two-dimensional (2-D) multislice sequence MR images, which are acquired in an interleaved way, are typically also corrupted with a slice-byslice constant intensity offset. This is commonly attributed to gradient eddy currents and crosstalk between slices [1]. In the literature, much research has been done on intraslice bias field correction, but the problem of such interslice artifacts has largely been ignored. A simple model for slice-by-slice offsets has been proposed in [17]. Every slice is assigned a constant multiplicative shading factor. Estimation of these offsets can be included in our algorithm by assigning to every slice $s$ a constant additive intensity term $a_{s}$ after logarithmic transformation. The expression to be minimized for single channel input data then becomes

$$
\begin{aligned}
-\sum_{s} \sum_{i \in s} \log _{e} & \left(\sum _ { j } G _ { \sigma _ { j } } \left(y_{i}-\mu_{j}\right.\right. \\
& \left.\left.-\left(\sum_{k} C_{k} \phi_{k}\left(x_{i}\right)+a_{s}\right)\right) p\left(\Gamma_{i}=j\right)\right)
\end{aligned}
$$

which means we have added a zero-order polynomial to each slice. As before, the polynomial $a_{s}$ is a weighted least-squares fit, but now only fitted to the voxels of slice $s$. Estimation of these $\left\{a_{s}\right\}$ forms another additional step in the algorithm.

However, we have noticed on multiple data sets that this model for the bias in 2-D multislice sequence MR images is too restrictive. We found, experimentally, that correcting for a 2-D polynomial bias field on each slice separately gives more 
consistent classifications. Therefore, we use the following model for this type of data sets:

$$
\begin{aligned}
-\sum_{s} \sum_{i \in s} \log _{e} & \left(\sum _ { j } G _ { \sigma _ { j } } \left(y_{i}-\mu_{j}\right.\right. \\
& \left.\left.-\sum_{k} C_{k_{s}} \phi_{k}\left(x_{i}\right)\right) p\left(\Gamma_{i}=j\right)\right)
\end{aligned}
$$

with $C_{s}=\left\{C_{k_{s}}\right\}$ the coefficients of the 2-D polynomial bias field on slice $s$. Such a slice-by-slice bias correction model was also used by Wells et al. [8] and Dawant et al. [6].

\section{Initialization with a Digital Brain Atlas}

The extended EM algorithm presented above iteratively interleaves classification, class distribution estimation and bias field correction. We initially set the bias field coefficients to zero and start the iterations by providing the algorithm an initial a priori estimate of the class probabilities $p\left(\Gamma_{i}=j\right)$ for each pixel $i$ and class $j$. This allows one to compute the class distribution parameters using (5) and (6) and to update the bias field parameters using (7), from which a new estimate for the classification can be derived using (2), etc.

The initial a priori pixel class probabilities are derived from a digital brain atlas, distributed with the SPM96 package [18] that contains spatially varying prior probability maps for the location of white matter, gray matter, and CSF, as shown in Fig. 1. These probability maps, with an isotropic spatial resolution of $2 \mathrm{~mm}$, were obtained by averaging binary white-matter, gray-matter and CSF segmentations of MR brain images from a large number of subjects, after normalization of all images into the same space using an affine transformation [19].

To apply the a priori information of the atlas, we first normalize the atlas to the space of the study image by matching the study image to a T1 template provided by SPM96 that is already coregistered with the atlas (see Fig. 1), using the affine multimodality registration technique based on maximization of mutual information of corresponding voxel intensities of Maes et al. [20]. It has been shown [20] that this registration criterion is fairly insensitive to moderate bias fields, such that it can be applied fully automatically and reliably to the uncorrected MR images. The class-specific distribution parameters $\boldsymbol{\mu}_{j}$ and $\Sigma_{j}$ are then computed from the study image, using the registered and reformatted a priori pixel class probability maps provided by the atlas. This approach frees us from having to interactively indicate representative pixels of each class, which makes our method more objective and reproducible and allows the method to be fully automated.

During subsequent iterations, the atlas is further used to spatially constrain the classification by setting the a priori class probability $p\left(\Gamma_{i}=j\right)$ in (2) equal to $p_{a t l a s}\left(\Gamma_{i}=j \mid x_{i}\right)$. Thus, the voxels are not only classified based on their intensities, but also based on their spatial position. This makes the algorithm more robust, especially when the images are corrupted with a heavy bias field.

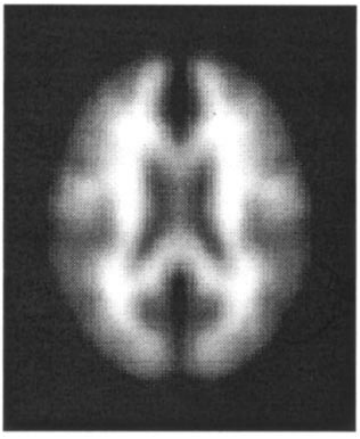

(a)

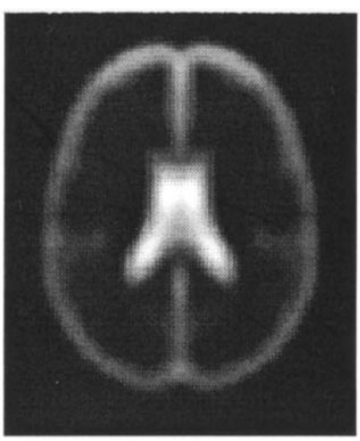

(c)

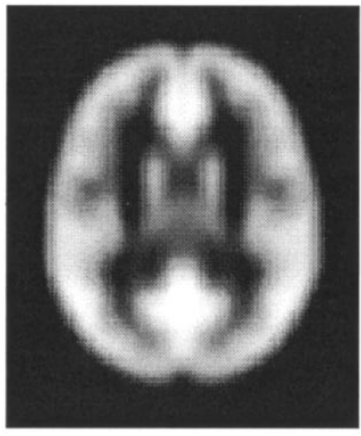

(b)

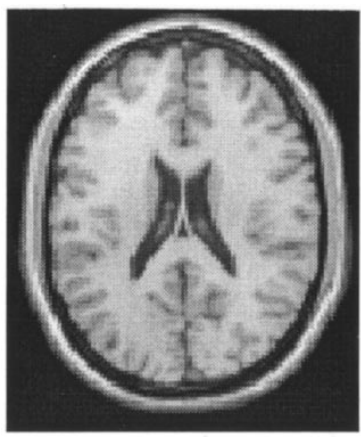

(d)
Fig. 1. Digital brain atlas with spatially varying a priori probability maps for (a) white matter, (b) gray matter, and (c) CSF. High intensities indicate high a priori probabilities. These maps were constructed by averaging binary segmentations of MR images from a large number of subjects, after affine spatial normalization [19]. (d) The atlas also contains a T1 template image which is used for registration of the study images to the space of the atlas.

\section{E. Implementation}

We implemented the algorithm as a modification of the Matlab-based [21] SPM96 [18] segmentation tool, which originally used the two-step classification algorithm without bias correction of Section II-A. The user can choose the maximum order of the polynomial bias field estimation, along with an option to search for a three-dimensional (3-D) bias field or for a 2-D field for each slice separately. There is no limit on the number of channels of the input data.

We use six classes: three brain tissue classes for white matter, gray matter, and CSF, respectively, two nonbrain tissue classes and one class for the background signal. Two classes are used for the nonbrain tissues because the intensity distribution of the nonbrain tissue voxels cannot be accurately modeled by a single Gaussian. Because the atlas provides $a$ priori probability maps for white matter, gray matter and CSF only, we construct a priori probability maps for the three other classes by subtracting the sum of the atlas probability maps from a map with value one in all entries and dividing the residual probability equally over the three other classes. Since we are only interested in brain tissue, we confine the algorithm to the region where the atlas indicates a nonzero $a$ priori probability for white matter, gray matter, or CSF. All other pixels are of no interest and are simply discarded.

In order to reduce calculation time, we estimate the parameters on down-sampled 3-D data and afterwards use those to perform bias estimation and tissue classification at full resolution. In order not to introduce extra partial volume 
voxels, we avoid interpolation during down sampling by only considering voxels lying on the original image grid. We start with a zero-order bias polynomial and increase the order when convergence is detected, until the maximum order, typically four, is reached. The iterations are stopped when the log likelihood no longer increases significantly (relative change smaller than $0.01 \%$ ), which is typically after about 30 iterations. The output of the program are the classifications in the form of probability images and an estimation of the bias field for each input channel.

When run on an IBM RS6000 workstation, the whole process of atlas registration, parameter estimation on downsampled 3-D data for a fourth-order polynomial, and subsequent classification of the full-resolution data typically takes about $0.5 \mathrm{~h}$ for a single-channel image with dimensions $256 \times$ $256 \times 52$. Increasing the size of the images and adding more channels increases the calculation time slightly.

\section{F. A 2-D Example}

Fig. 2 illustrates the bias correction algorithm presented above, with the simple example of a single sagittal slice of a T1-weighted MR image of the head. The figure shows the status of the algorithm on the last iteration. Based on the bias and distribution estimation from the previous iteration, a classification is made. The predicted signal without bias [Fig. 2(b)] is calculated from this classification and from the distribution estimation. The residue image [Fig. 2(c)] is obtained by subtracting this predicted signal from the original image. The bias [Fig. 2(e)] is estimated as the weighted least squares fit through the residue image, using the weights shown in Fig. 2(d).

The residue and weight images are the key to understanding the algorithm. If our model had been perfect, then the residue image would be the bias field corrupted with some noise. However, it can be seen that this is not true, especially not for CSF and the tissues surrounding the brain. The reason is that, unlike white matter and gray matter, the intensity distribution of those classes is not very peaked. When a Gaussian distribution model is used, the mean may be unrepresentative for the intensity of the pixels belonging to those classes, introducing large errors in the residue image.

However, the influence of such errors on the estimation of the bias field is tempered by the weights. Indeed, classes whose distributions are not very peaked and, as a consequence, show large errors in the residue image, will also have a large variance. Since the weights are inversely proportional to the variance, those classes will be assigned a low weight for bias estimation. This can be seen in Fig. 2(d). Pixels belonging to white matter and, to a lesser extent, gray matter have a very high weight compared to pixels belonging to other classes. Thus, the bias field is estimated mainly from brain tissue pixels and extrapolated via the polynomial model to regions where the bias field cannot be confidently estimated.

\section{VALIDATION}

Quantitative validation of the performance of image analysis methods on clinical data is difficult due to the lack of ground

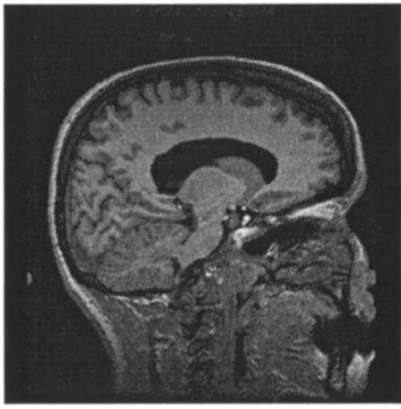

(a)

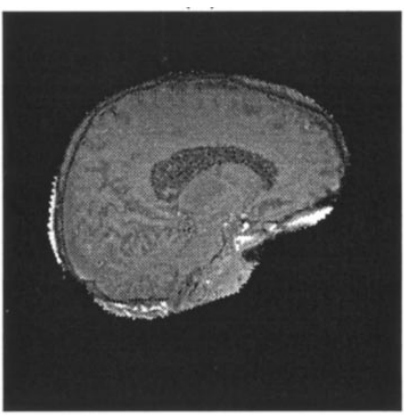

(c)

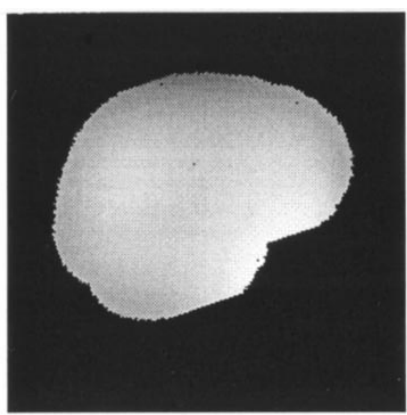

(e)

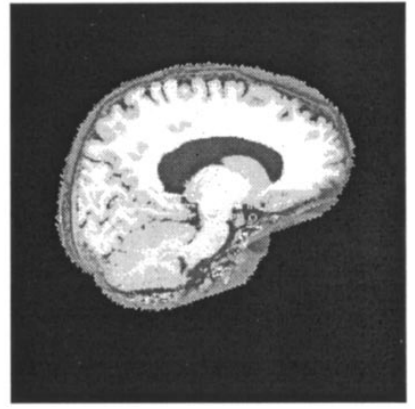

(b)

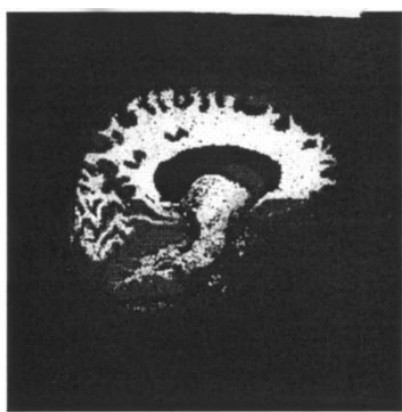

(d)

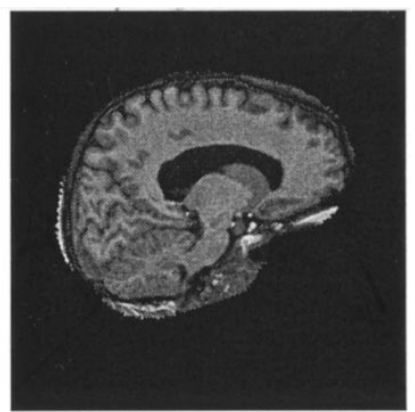

(f)
Fig. 2. Illustration of the bias correction algorithm on a 2-D T1-weighted MR image at the last iteration. (a) Original image. (b) Predicted signal based on previous iterations. (c) Residue image. (d) Weights. (e) Estimated bias field. (f) Corrected image. The algorithm is confined to the region where the atlas indicates a nonzero a priori probability for white matter, gray matter, or CSF.

truth. We therefore evaluated the performance of the bias correction algorithm on simulated T1-, T2-, and PD-weighted MR images corrupted with a known bias field, as shown in Fig. 3. These images were obtained from the BrainWeb MR simulator [22]-[24]. The images have an isotropic voxelsize of $1 \times 1 \times 1 \mathrm{~mm}$ and are corrupted by a bias field of $40 \%$. We varied the standard deviation of the noise between $1-9 \%$ of the mean white matter intensity. For each noise level, we used the three-step EM algorithm, as presented above, to estimate the bias fields for the different channels from each channel separately (single channel input), as well as from all channels combined (three-channel input). The estimated and the imposed bias fields were compared by dividing them and calculating the coefficient of variation (variance divided by mean times 100) of the resulting image. This coefficient is zero if the true bias field has been totally recovered. 

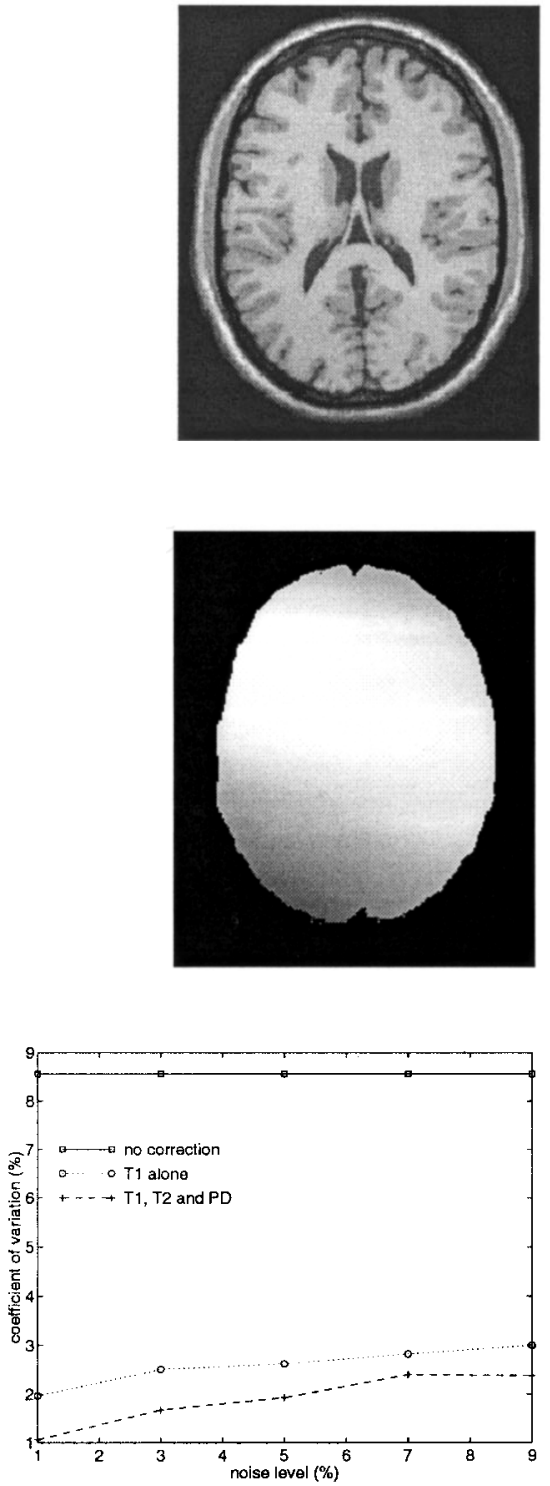
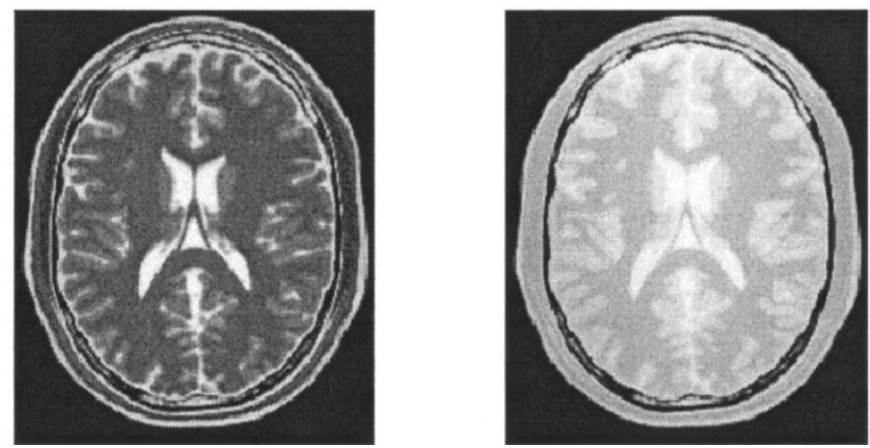

(a)

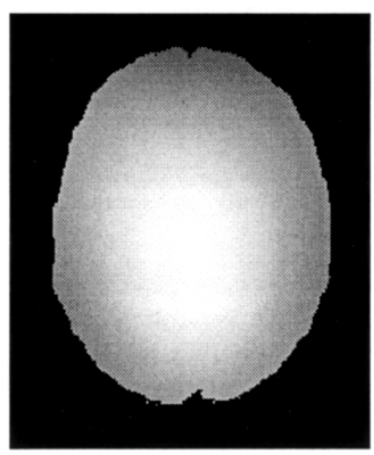

(b)
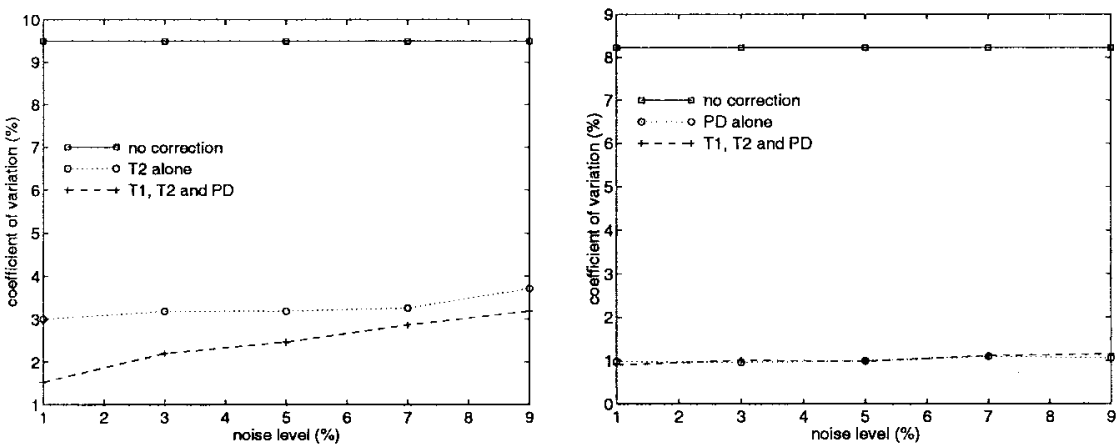

(c)

Fig. 3. Validation of the bias correction algorithm on simulated data. (a) Simulated T1-, T2-, and PD-weighted MR images obtained from the BrainWeb simulator [22]. (b) Known bias fields imposed on the T1-, T2-, and PD-weighted images. The amplitude of the bias fields is exaggerated for visualization purposes. (c) Coefficient of variation before correction, after single-channel correction and after three-channel correction for different noise levels.

The plots in Fig. 3 show the coefficient of variation for the T1-, T2-, and PD-weighted images before and after correction. It can be seen that, in all cases, the algorithm has significantly reduced the bias field. Adding noise decreases the accuracy of the bias estimation only slightly. For the T1- and T2-weighted images, the performance is clearly better when all the channels are combined, compared to the situation where only a single channel is used. This can be explained by considering that each channel provides additional information, yielding better classifications and, thus, more accurate bias estimations.

\section{RESULTS}

In this section we describe experimental results on various MR images of the head. We applied the algorithm to correct the bias field on images with different resolution, number of channels and severity of field inhomogeneity. In all the experiments, we used a fourth-order polynomial.

\section{A. Single-Channel Data}

Fig. 4 shows the classification result for a high-resolution sagittal T1-weighted MR image (Siemens Vision 1.5 T, 3-D MPRAGE, $256 \times 256$ matrix, $1.25-\mathrm{mm}$ slice thickness, 128 slices, $\mathrm{FOV}=256 \mathrm{~mm}, \mathrm{TR}=11.4 \mathrm{~ms}, \mathrm{TE}=4.4 \mathrm{~ms}$ ) obtained with the two-step classification algorithm without bias correction of Section II-A and with our extended algorithm using a 3-D polynomial bias field model. Because a relatively strong bias field reduces the intensities at the top of the head, the twostep algorithm wrongly classifies white matter in that area as gray matter. The extended algorithm succeeds in compensating for this and provides better segmentations. 
(a)

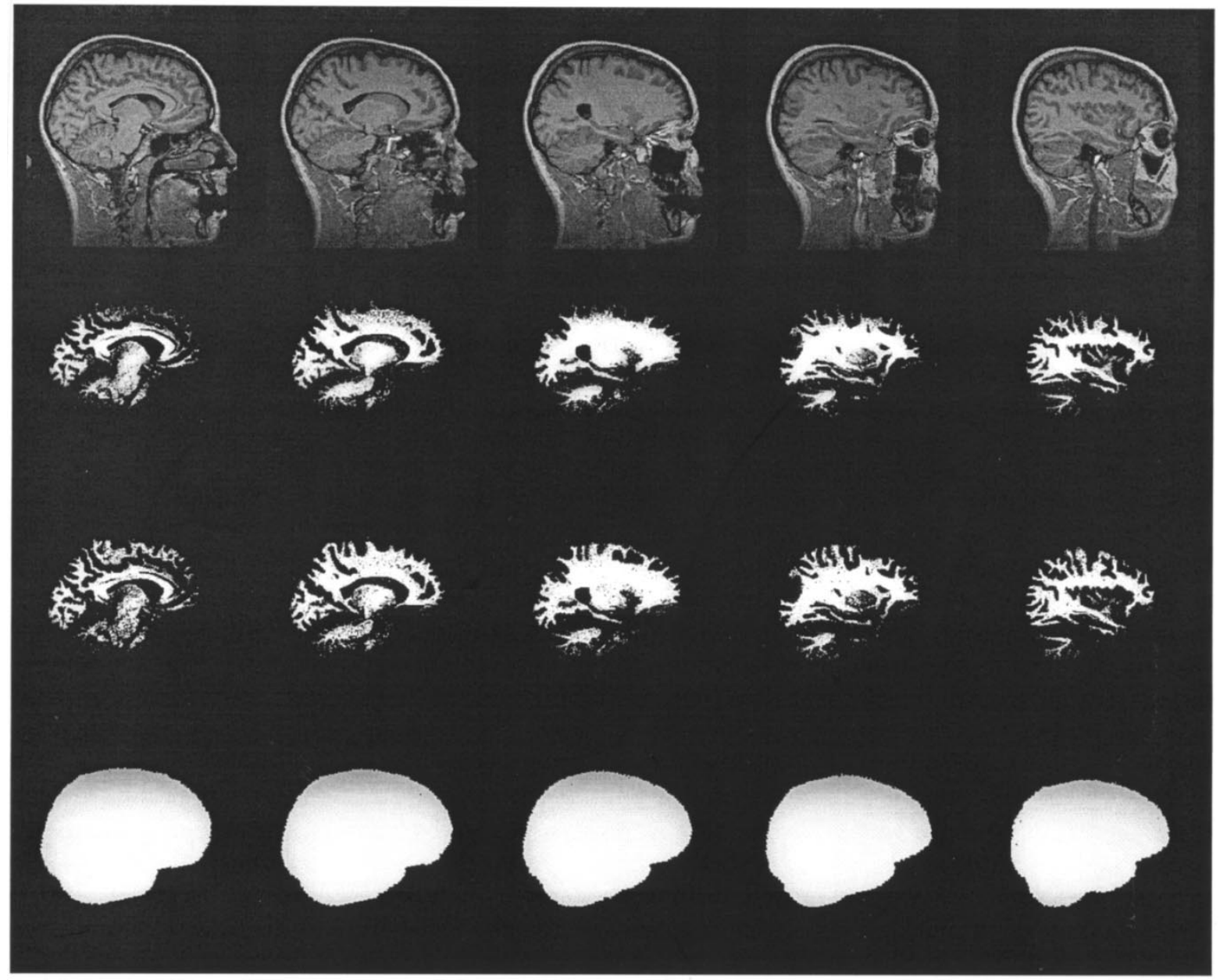

Fig. 4. Slices of a high-resolution T1-weighted MR image illustrating the performance of the method on single-channel data. (a) Original data. (b) White-matter classification with the two-step classification algorithm without bias correction. (c) White-matter classification with our three-step algorithm with bias correction. (d) Estimated bias field.

Fig. 5 shows 3-D rendered images of the white- and graymatter segmentation of this data set. It can be seen that without bias correction, gray matter is a little bit thinner at the top of the brain and white matter is seriously misclassified as gray matter.

\section{B. Multichannel Data}

Fig. 6 shows an example of two-channel input data consisting of historical PD and T2-weighted MR images (GE Signa $1.5 \mathrm{~T}, 40$ axial slices of $256 \times 256$, pixel size of $0.94 \times 0.94 \mathrm{~mm}^{2}, 5-\mathrm{mm}$ slice thickness, 2-mm interslice gap) taken from a schizophrenia study. Although the effect of the bias field is hardly visible, it has a big impact on the resulting segmentation. For visualization purposes, we derived a hard segmentation from the probability maps produced by the algorithm by assigning each voxel exclusively to the class it most probably belongs to. At the top and the bottom of the images, the image intensities are slightly reduced due to the bias field such that, without bias correction, gray matter is wrongly classified as white matter. After bias correction, the segmentation is clearly improved.
A quantitative analysis of the bias correction for this example is shown in Table I. We manually selected some representative pixels for white and gray matter and calculated the coefficient of variation of their intensities (standard deviation divided by the mean) before and after bias correction. As is clear from Table I, the coefficient of variation has decreased significantly after bias correction. The remaining variance can be attributed to tissue microtexture, noise, partial volume effect, and remaining bias.

The effect of bias correction can also be visualized in feature space. Fig. 7 shows scatter plots of PD versus T2 intensity of the selected white- and gray-matter pixels before and after bias correction. Clearly, the bias field causes a serious overlap in feature space, which leads to severe misclassifications. After bias correction, white-matter and gray-matter pixels are much more clustered and their clusters are also more separated, such that better classifications can be made.

\section{2-D Multislice Sequence}

To illustrate bias correction for images with slice-by-slice constant offsets, we have processed the single-channel 2-D multislice T1-weighted image shown in Fig. 8 (19 sagittal 
TABLE I

CoEfFicient of VARIATION For the Data of Fig. 6

\begin{tabular}{c|c|rr|rr}
\hline & & \multicolumn{2}{|c|}{ T2 } & \multicolumn{2}{c}{ PD } \\
\hline \multicolumn{1}{c|}{ class } & samples & original & corrected & original & corrected \\
\hline white matter & 1744 & $9.47 \%$ & $6.76 \%$ & $5.65 \%$ & $3.45 \%$ \\
gray matter & 925 & $11.18 \%$ & $8.03 \%$ & $9.64 \%$ & $5.56 \%$ \\
\hline
\end{tabular}

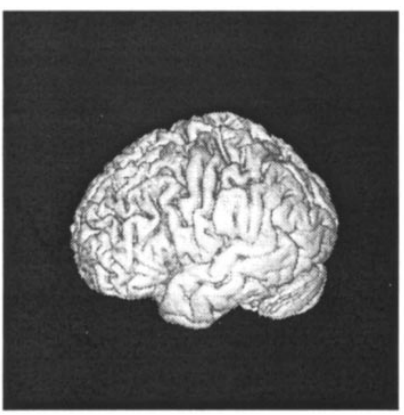

(a)

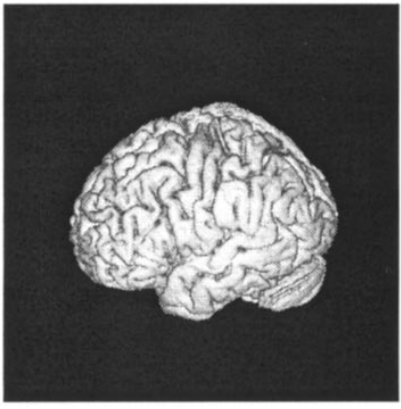

(c)

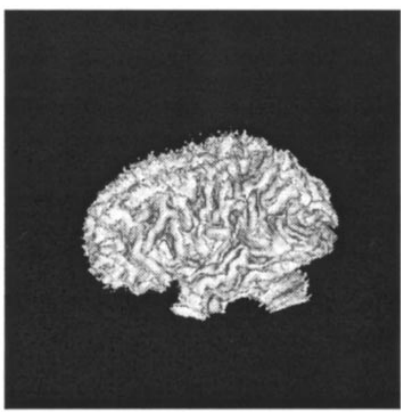

(b)

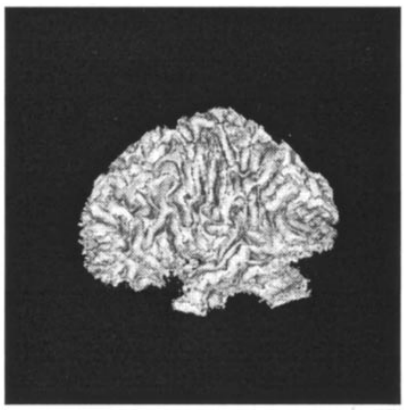

(d)

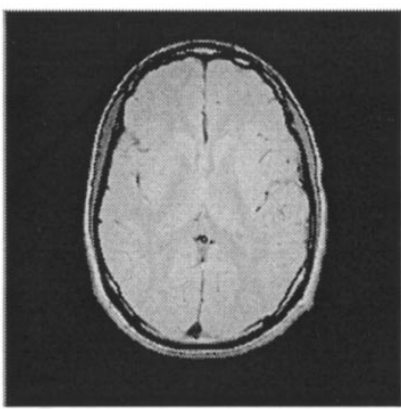

(a)

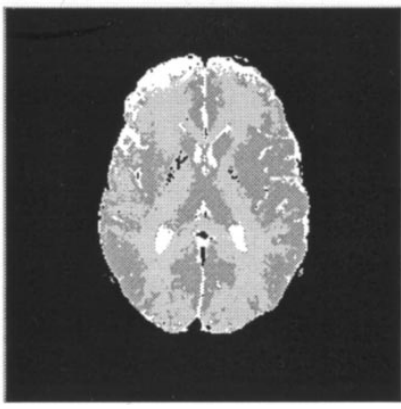

(c)

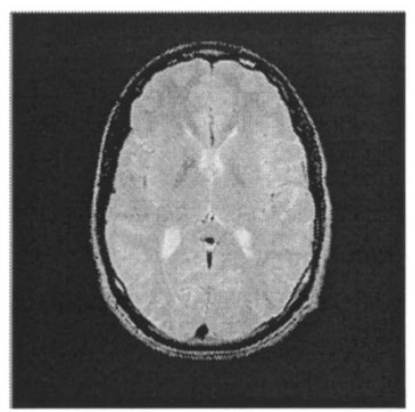

(b)

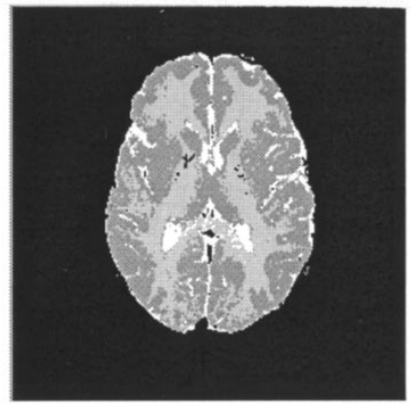

(d)

Fig. 5. 3-D rendering of the gray-matter (left) and white-matter (right) segmentation of the MR image of Fig. 4. (a) and (b) Two-step algorithm without bias correction. (c) and (d) Three-step algorithm with bias correction.

slices of $256 \times 256$, voxel size of $0.94 \times 0.94 \times 5.00$ $\mathrm{mm}^{3}$, interslice gap of $2.00 \mathrm{~mm}$ ). The slice-by-slice offsets can clearly be seen as an interleaved bright-dark intensity pattern in a cross section orthogonal to the slices. The estimated bias and the corrected images are also shown. It can be seen that the algorithm compensates for the slice-by-slice offsets and produces more consistent classification results.

\section{Surface Coil Image}

Fig. 9 shows a T1-weighted 2-D multislice image acquired with a surface coil (Siemens Vision 1.5 T, 20 sagittal slices of $256 \times 256$, voxel size of $0.90 \times 0.90 \times 7.20 \mathrm{~mm}^{3}$, $1.20-\mathrm{mm}$ interslice gap). Despite their limited region of detection, surface coils can be used in situations where the improved signal-to-noise ratio and the higher spatial resolution are beneficial: for instance, for functional imaging. An important drawback is the large bias field, as is clear from Fig. 9, which impedes even visual inspection by a human observer.

Nevertheless, our algorithm performs very well on such images, as is apparent from Fig. 9. After bias correction, white and gray matter have the same intensity all over the image, making visual inspection possible. This example illustrates

Fig. 6. Segmentation of multichannel PD- and T2-weighted MR data. (a) PD slice. (b) T2 slice. (c) Segmentation with the two-step algorithm without bias correction. (d) Segmentation with our three-step algorithm with bias correction. Tissue classes are color-coded as follows: bright $=\mathrm{CSF}$; dark gray $=$ gray matter; light gray $=$ white matter; black $=$ background and nonbrain tissues.

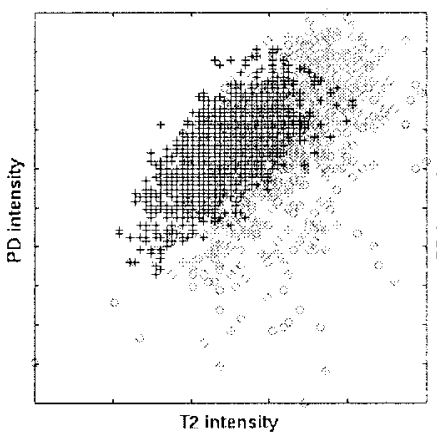

(a)

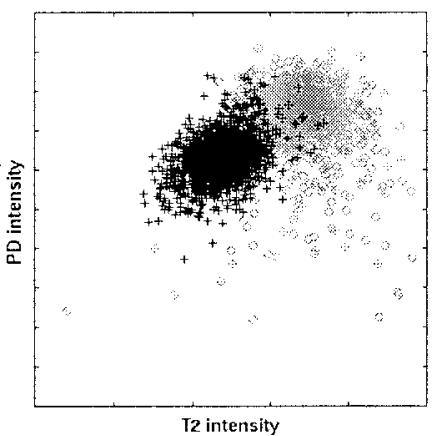

(b)
Fig. 7. Separation of white matter (crosses) and gray matter (circles) in feature space. The plots show the intensity of T2 versus the intensity of PD before (left) and after (right) bias correction for the same points used in Table I.

the robustness of our algorithm, even for fairly large bias fields. However, it should be pointed out that in this case the procedure was not completely automatic. Although the intensity based registration algorithm we use to normalize the 

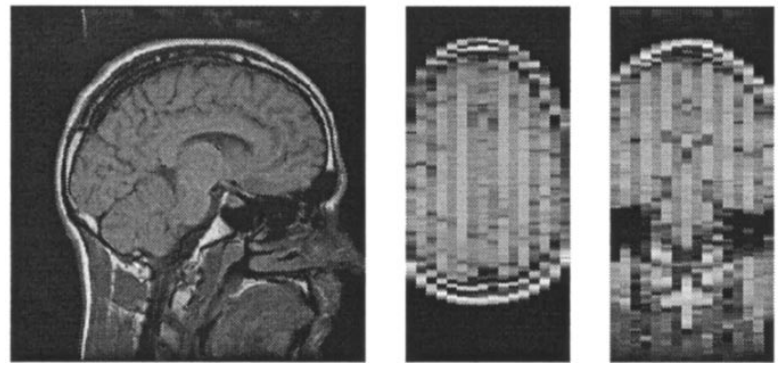

(a)
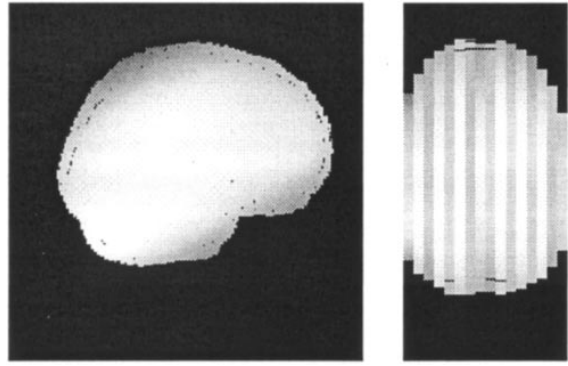

(b)
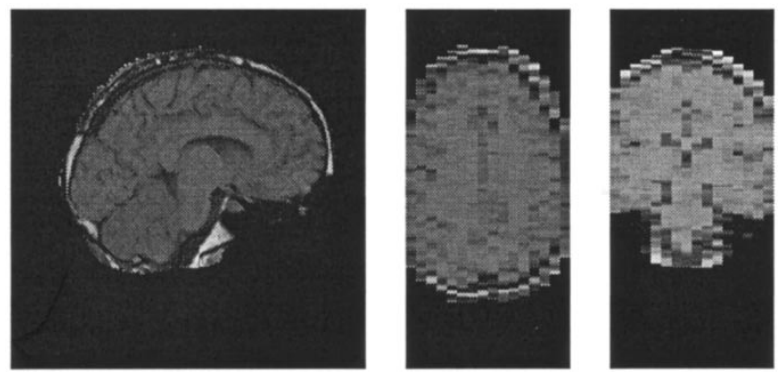

(c)
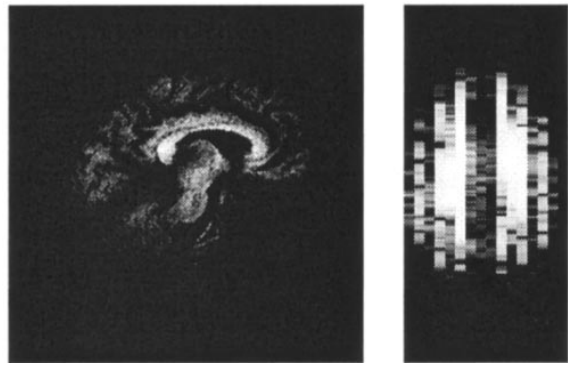

(d)
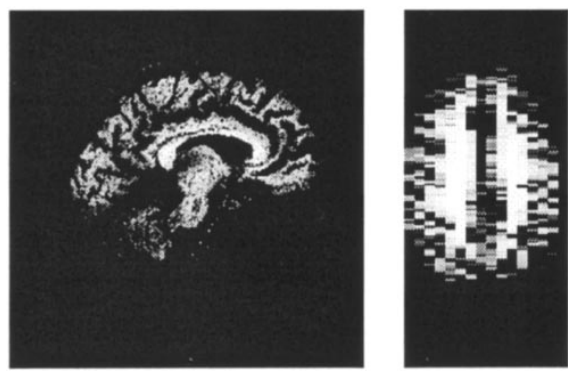

(e)

Fig. 8. An example of bias correction of a T1-weighted 2-D multislice image corrupted with slice-by-slice offsets. (a) Original data. (b) Estimated bias. (c) Corrected data. (d) White matter probability map for the two-step algorithm (no bias correction). (e) White matter probability map for our three-step algorithm.
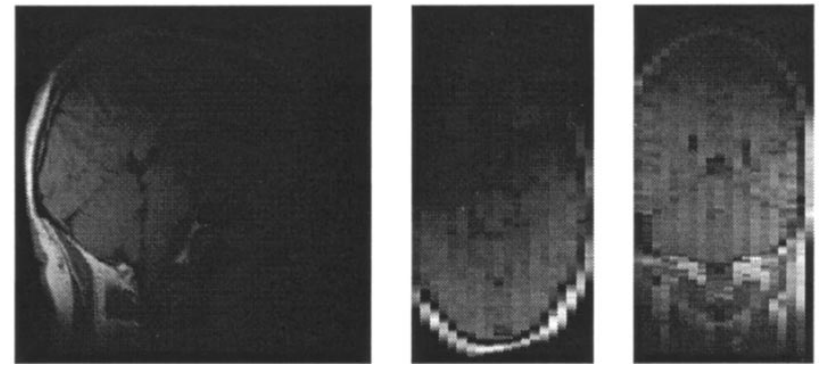

(a)
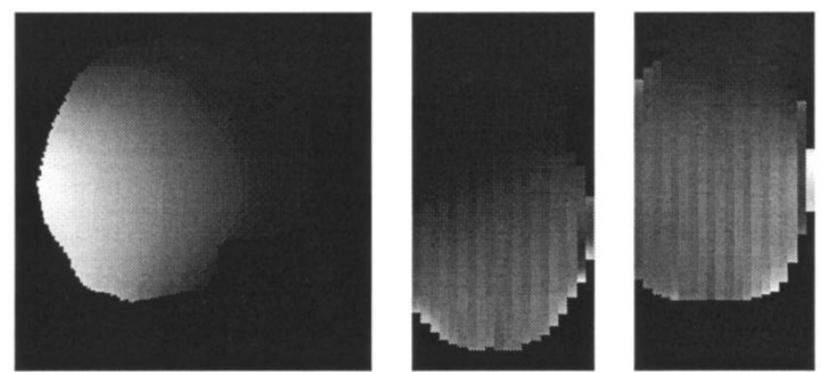

(b)
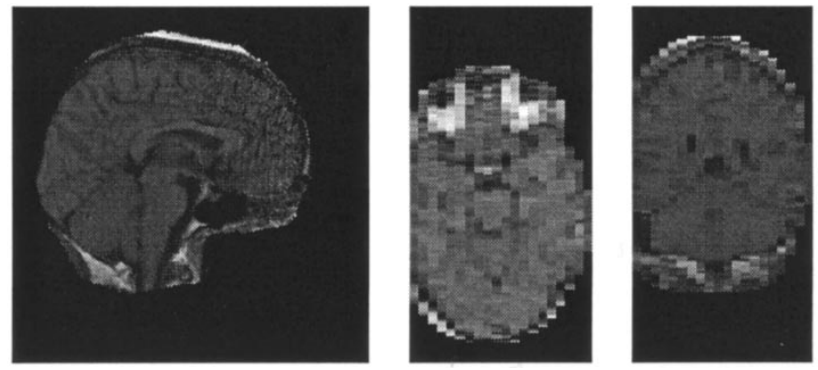

(c)

Fig. 9. The 3-step algorithm applied to a T1-weighted surface coil image (a) Original data. (b) Estimated bias. (c) Corrected data.

atlas to the study images is fairly insensitive to moderate bias fields, it was confused by the very large bias field in this image such that we had to provide a good initial position manually to make the registration work.

\section{DISCUSSION}

The MR bias correction method we proposed in this paper models the MR signal as the realization of a random process with a parametric probability density, corrupted by a smooth parametric bias field. We presented an extended EM algorithm that interleaves tissue classification with class distribution and bias field parameter estimation, increasing the likelihood of the model parameters at each iteration. The main characteristics of our algorithm are the weights used to estimate the parameters of the bias field model, iterative reestimation of the tissue class distributions, and the use of a priori tissue probability maps from a digital brain atlas.

The bias field is modeled as a linear combination of smooth basis functions. The parameters of this model are computed from a weighted least-squares fit to the residue image, each pixel's weight being inversely proportional to the variance of the class that pixel belongs to. The bias field is therefore 
computed primarily from pixels that belong to classes with a narrow intensity distribution, such as white and gray matter. For these classes, the residue image is a good indicator for the bias field, while for CSF and nonbrain tissue classes that have a rather broad intensity distribution, the residue image shows large errors, as can be seen in Fig. 2. Our algorithm is robust for such errors because it automatically assigns a low weight to these regions. The smooth spatial model of the bias field extrapolates the bias field from regions where it can be confidently estimated from the data (white and gray matter) to regions where such an estimate is ill conditioned (CSF, nonbrain tissues).

The algorithm of Wells et al. [8] uses the same residue and weights as our algorithm, but they compute the bias field from the residue image by spatial filtering. Because large filter sizes are required for sufficient smoothing, this approach can suffer significantly from boundary effect artifacts due to the limited extent of the image domain [25]. Moreover, the bias estimation is a mere low-pass filtered version of the residue in large regions with the same tissue class, independent of the weights. In regions with low weights, such as the ventricular area in Fig. 2, this can be expected to lead to errors.

We used a fourth-order polynomial model for the bias field [3], [7], [26]. We have not investigated other representations such as splines [6], [27], [28], nor did we investigate techniques to estimate the order or the stiffness of the bias field model automatically from the data itself. For 2-D multislice sequence images, we found that correcting for a 2-D polynomial bias field on each slice separately, which was also done in [6] and [8], yields a more consistent classification than when a single 3-D polynomial is used, even when correcting for slice-by-slice constant intensity offsets between slices.

Our extended EM algorithm reestimates at each iteration the parameters $\boldsymbol{\mu}_{j}$ and $\boldsymbol{\Sigma}_{j}$ of the normal intensity distribution of each class, while in earlier approaches [8], [10] these parameters were determined by manual training and kept fixed during iterations. It has been reported [10], [26], [29] that Wells' method [8] is sensitive to the training of its classifier: the algorithm produces different results depending on which pixels were selected for training. Moreover, pixels the algorithm was not trained for may cause severe errors in the residue image and the bias field estimation [10], [29]. This typically occurs in regions surrounding the brain, consisting of several different tissue types that are easily overlooked during training. Guillemaud and Brady [10] proposed to account for this by modeling these nonbrain tissues by a single class with a uniform distribution, artificially assigning the nonbrain tissue pixels a zero weight for the bias estimation. This is not necessary with our algorithm. The class distribution parameters are updated at each iteration from all pixels in the image and classes consisting of different tissue types are automatically assigned a large variance and, thus, a low weight for bias estimation.

Our algorithm avoids manual training of the classifier by using a digital brain atlas that provides a priori information about the expected location of white matter, gray matter, and CSF. This yields objective and reproducible results and allows full automation of the algorithm. Moreover, the use of an atlas makes our algorithm significantly more robust. Even for severe bias fields as in Fig. 9, the method converges to the right solution because it is forced by the atlas to classify most of the pixels correctly. A drawback of this approach is that it assumes such an atlas to be available, which currently limits the practical use of our method to brain images only. Also, while the registration approach is able to compensate for global differences in brain morphology, for instance when segmenting MR images of children [12], we found that the method may occasionally fail, due to poor initialization in the presence of large pathology-induced abnormalities in brain shape, for instance, in subjects with dramatically increased ventricles. This problem may be overcome by using nonrigid rather than affine registration for atlas matching.

In our current implementation, the atlas is normalized to the study image using an affine geometric transformation which is computed automatically from the data [20]. The registration to the atlas may fail for surface coil images with very large bias, such that manual intervention is sometimes required to provide good initial alignment. The fuzziness of the atlas we are currently using helps to temper the influence of local misregistration, not corrected for by the affine registration. However, while the unsharpness of the atlas has no significant influence on the bias correction itself, it may occasionally introduce voxel classification errors. Because some nonbrain voxels may be assigned a nonzero a priori brain tissue probability by the atlas, they can be misclassified as white or gray matter. Inclusion of contextual constraints on the classification and elastic registration techniques may overcome this problem and are currently being investigated [12], [30].

A number of authors have proposed bias correction methods that do not use an intermediate classification [26], [27]. The approach of Sled et al. [27], for instance, is based on deconvolution of the histogram of the measured signal, assuming that the histogram of the bias field is Gaussian, while Brechbühler et al. [26] find the bias field that optimizes some heuristic measure for the sharpness of the histogram of the corrected image. Contrary to our approach, these methods treat all pixels alike for bias estimation. This looks rather unnatural, since it is obvious that the white-matter pixels, which have a narrow intensity histogram, are much more suited for bias estimation than, for instance, the tissues surrounding the brain or ventricular CSF. As argued above, our algorithm takes this into account, explicitly, by the class-dependent weights assigned to each pixel.

\section{CONCLUSION}

In this paper, we presented a method for MR bias field correction and tissue classification using a priori tissue probability maps from a digital brain atlas. Our algorithm is fully automated, avoids user interaction, and therefore yields objective and reproducible results. We extended a two-step EM classification algorithm without bias correction to a threestep procedure, incorporating an explicit parametric model of the bias field. The algorithm interleaves classification with parameter estimation, yielding better results at every iteration. 
Results were presented and discussed on various MR data sets with important field inhomogeneities and slice-by-slice offsets. We related the algorithm to some previously published bias correction algorithms. Future work will focus on incorporating elastic registration techniques to normalize the atlas to the space of the images and on validation of the tissue classification algorithm.

\section{ACKNOWLEDGMENT}

The authors wish to acknowledge the contribution of $\mathbf{J}$. Michiels and H. Bosmans.

\section{REFERENCES}

[1] A. Simmons, P. S. Tofts, G. J. Barker, and S. R. Arridge, "Sources of intensity nonuniformity in spin echo images at $1.5 \mathrm{~T}$," Magn. Resonance Med., vol. 32, pp. 121-128, 1994.

[2] J. G. Sled and G. B. Pike, "Understanding intensity nonuniformity in MRI," in Proc. Medical Image Computing Computer-Assisted Intervention-MICCAI'98 (Lecture Notes in Computer Science). Berlin, Germany: Springer-Verlag, 1998, vol. 1496, pp. 614-622.

[3] M. Tincher, C.R. Meyer, R. Gupta, and D. M. Williams, "Polynomial modeling and reduction of RF body coil spatial inhomogeneity in MRI," IEEE Trans. Med. Imag., vol. 12, pp. 361-365, June 1993.

[4] S. E. Moyher, D. B. Vigneron, and S. J. Nelson, "Surface coil MR imaging of the human brain with an analytic reception profile correction," $J$. Magn. Resonance Imag., vol. 5, pp. 139-144, Mar./Apr. 1995.

[5] R. C. Gonzalez and R. E. Woods, Digital Image Processing. Reading, MA: Addison-Wesley, 1993

[6] B. M. Dawant, A. P. Zijdenbos, and R. A. Margolin, "Correction of intensity variations in MR images for computer-aided tissue classification," IEEE Trans. Med. Imag., vol. 12, pp. 770-781, Dec. 1993.

[7] C. R. Meyer, P. H. Bland, and J. Pipe, "Retrospective correction of MRI amplitude inhomogeneities," in Proc. First Int. Conf. Computer Vision, Virtual Reality, Robotics Medicine (CVRMED'95) (Lecture Notes in Computer Science), N. Ayache, Ed. Berlin, Germany: Springer-Verlag, 1995, vol. 905, pp. 513-522.

[8] W. M. Wells, III, W. E. L. Grimson, R. Kikinis, and F. A. Jolesz, "Adaptive segmentation of MRI data," IEEE Trans. Med. Imag., vol. 15, pp. 429-442, Aug. 1996

[9] A. P. Dempster, N. M. Laird, and D. B. Rubin, "Maximum likelihood from incomplete data via the EM algorithm," J. R. Stat. Soc., vol. 39, pp. $1-38,1977$.

[10] R. Guillemaud and M. Brady, "Estimating the bias field of MR images," IEEE Trans. Med. Imag., vol. 16, pp. 238-251, June 1997.

[11] K. Held, E. Rota Kops, B. J. Krause, W. M. Wells III, R. Kikinis, and H.-W. Müller-Gärtner, "Markov random field segmentation of brain MR images," IEEE Trans. Med. Imag., vol. 16, pp. 878-886, Dec. 1997.

[12] K. Van Leemput, F. Maes, D. Vandermeulen, and P. Suetens, "Automated model-based tissue classification of MR images of the brain," IEEE Trans. Med. Imag., (Special Issue on Model-Based Analysis of Medical Images), vol. 18, pp. 897-908, Oct. 1999.

[13] Z. Liang, J. R. MacFall, and D. P. Harrington, "Parameter estimation and tissue segmentation from multispectral MR images," IEEE Trans. Med. Imag., vol. 13, pp. 441-449, Sept. 1994.
[14] C. F. J. Wu, "On the convergence properties of the EM algorithm," Ann. Stat., vol. 11, no. 1, pp. 95-103, 1983

[15] J. A. Hartigan, Clustering Algorithms. New York: Wiley, 1975, ch. 5, pp. 113-129.

[16] S. Patz and H. Gudbjartsson, "The Rician distribution of noisy MRI data," Magn. Resonance Med., vol. 34, pp. 910-914, 1995.

[17] A. P. Zijdenbos, B. M. Dawant, and R. A. Margolin, "Intensity correction and its effect on measurement variability in the computer-aided analysis of MRI," in Proc. 9th Int. Symp. Exhibition Computer Assisted Radiology (CAR'95). Berlin, Germany: Springer-Verlag, pp. 216-221.

[18] J. Ashburner, K. Friston, A. Holmes, and J.-B. Poline, Statistical Parametric Mapping, The Wellcome Dept. Cognitive Neurology, Univ. College London, London, U.K. Available: http://www.fil.ion.ucl.ac.uk/spm/

[19] A. C. Evans, D. L. Collins, S. R. Mills, E. D. Brown, R. L. Kelly, an T. M. Peters, "3D statistical neuroanatomical models from 305 MRI volumes," in Proc. IEEE Nuclear Science Symp. Medical Imaging Conf., 1993, pp. 1813-1817.

[20] F. Maes, A. Collignon, D. Vandermeulen, G. Marchal, and P. Suetens, "Multi-modality image registration by maximization of mutual information," IEEE Trans. Med. Imag., vol. 16, pp. 187-198, Apr. 1997.

[21] Matlab. Natick MA: The MathWorks Inc.

[22] C. A. Cocosco, V. Kollokian, R. K.-S. Kwan, and A. C. Evans, "BrainWeb: Online interface to a 3D MRI simulated brain database," NeuroImage, vol. 5, no. 4 pt. 2/4 S425, 1997. Available: http://www.bic.mni.mcgill.ca/brainweb/

[23] R. K.-S. Kwan, A. C. Evans, and G. B. Pike, "An extensible MRI simulator for post-processing evaluation," in Proc. Visualization in Biomedical Computing - VBC'96 (Lecture Notes in Computer Science). Berlin, Germany: Springer-Verlag, 1996, vol. 1131 pp. 135-140.

[24] D. L. Collins, A. P. Zijdenbos, V. Kollokian, J. G. Sled, N. J. Kabani, C. J. Holmes, and A. C. Evans, "Design and construction of a realistic digital brain phantom," IEEE Trans. Med. Imag., vol. 17, pp. 463-468, June 1998.

[25] J. G. Sled, "A nonparametric method for automatic correction of intensity nonuniformity in MRI data," M.S. thesis, Dept. Biomedical Eng., McGill Univ., Montréal, P.Q., Canada, May 1997.

[26] C. Brechbühler, G. Gerig, and G. Székely, "Compensation of spatial inhomogeneity in MRI based on a parametric bias estimate," in Proc. Visualization in Biomedical Computing (VBC '96) (Lecture Notes in Computer Science). Berlin, Germany: Springer-Verlag, 1996, vol. 1131, pp. 141-146.

[27] J. G. Sled, A. P. Zijdenbos, and A. C. Evans, "A nonparametric method for automatic correction of intensity nonuniformity in MRI data," IEEE Trans. Med. Imag., vol. 17, no. 1, pp. 87-97, Feb. 1998.

[28] S. Gilles, M. Brady, J. Declerck, J.-P. Thirion, and N. Ayache, "Bias field correction of breast MR images," in Proc. Visualization in Biomedical Computing (VBC '96) (Lecture Notes in Computer Science). Berlin, Germany: Springer-Verlag, 1996, vol. 1131, pp. 153-158

[29] J. G. Sled, A. P. Zijdenbos, and A. C. Evans, "A comparison of retrospective intensity nonuniformity correction methods for MRI," in Proc. XVth Int. Conf. Information Processing in Medical Imaging (IPMI'97) (Lecture Notes in Computer Science). Berlin, Germany: Springer-Verlag, 1997, pp. 459-464.

[30] T. Gaens, F. Maes, D. Vandermeulen, and P. Suetens, "Non-rigid multimodal image registration using mutual information," in Proc. First Int Conf. Medical Image Computing and Computer-Assisted Intervention (MICCAI'98) (Lecture Notes in Computer Science) William M. Wells, Alan Colchester, and Scott Delp, Eds. Cambridge, MA: SpringerVerlag, 1998, vol. 1496, pp. 1099-1106. 